\title{
FRACTURE HEALING ASSESSMENT BASED ON IMPACT TESTING: IN VITRO SIMULATION AND MONITORING OF THE HEALING PROCESS OF A TIBIAL FRACTURE WITH EXTERNAL FIXATOR
}

\author{
FRANCESCA DI PUCCIO \\ Department of Civil and Industrial Engineering, University of Pisa, Largo Lazzarino, \\ Pisa, IT 56122 Italy \\ dipuccio@ing.unipi.it \\ LORENZA MATTEI \\ Department of Civil and Industrial Engineering, University of Pisa, Largo Lazzarino, \\ Pisa, IT 56122 Italy \\ l.mattei@ing.unipi.it \\ ANTONIA LONGO \\ Department of Civil and Industrial Engineering, University of Pisa, Largo Lazzarino, \\ Pisa, IT 56122 Italy \\ antonia.longo1@gmail.commailto:1.mattei@ing.unipi.it \\ STEFANO MARCHETTI \\ Department of Translational Research and of New Surgical and Medical Technologies, University of Pisa, \\ Pisa, IT 56122 Italy \\ stefano.marchetti@unipi.it \\ Received Day Month Year \\ Revised Day Month Year
}

The healing process of bone fractures is monitored with X-rays or manipulation, so its assessment is subjective and depends on the skills of the operator. Alternative and quantitative approaches are under investigation. They are generally based on the estimation of bone stiffness that is known to increase with the healing process.

The application of experimental modal analysis to fracture healing assessment is investigated in this study focusing on fractures treated with an external fixator. The aim is to prove the capability of this approach to detect changes in the bone stiffness (or bone-callus stiffness). In vitro tests were performed on a tibia phantom where the healing process was simulated creating three different types of callus surrogates, using glue and resin. The resonant frequencies of the phantom with screwed pins and of the phantom with the complete fixator were estimated. Results indicated, as expected, an increase in the frequencies with the stiffness of the simulated callus. This study encourages the application of experimental modal analysis to fracture healing monitoring, taking advantage of pins and external fixator. The clinical application of this approach can offer remarkable advantages with respect to the actual approach based on X-rays and manipulation. In addition to being less invasive, it can provide quantitative indications, against the current qualitative assessment.

Keywords: Fracture healing; experimental modal analysis; external fixator. 


\section{Introduction}

External fixation is a well-established procedure for the treatment of long bone fractures, known also by Hippocrate [Pontarelli, 1982, Checketts and Young, 2003]. Modern fixators are capable of being adapted to different and complex cases both to open and closed fractures [Bhandari et al., 2001]. A successful use of these devices requires the correct timing for their dynamization and removal, adjusted to the evolution of the healing process. In fact, re-fractures can occur in unripe dynamization [Checketts and Young, 2003], while a delayed timing means unnecessary prolonged and costly treatments and possible pin related problems [Davies et al., 2005].

In clinical practice, bone healing assessment is based on X-rays and manipulation. It is thus affected by two main limitations: firstly, it is subjective and depends on the skills of the operator and secondly, it cannot be performed as often as may be clinically desirable, e.g. every two weeks. Alternative and less invasive solutions are thus being sought. Different approaches have been proposed in the literature since the Nineties, e.g. [Cunningham et al., 1990, Markel and Chao, 1993], ranging from ultrasound [Li et al., 2013] to angle measurement [Eastaugh-Waring et al., 2000]. However, none of them has been considered reliable enough to replace $\mathrm{X}$-rays in clinical practice.

Recently, the present authors proposed a procedure based on mechanical vibrations to evaluate the stiffness of a tibia phantom treated with an external fixator [Mattei et al., 2017]. The application of mechanical vibrations and modal analysis to fracture healing assessment is not new in the literature, e.g. [Cunningham et al., 1990]. The main idea behind this approach is that during the healing process the bone stiffness increases and this variation affects also the natural frequencies of the bone and of the limb. Its application was restricted due to the complexities of hardware and software tools (originally oscilloscopes were used). Practical difficulties were also due to the damping effect of soft tissues, because both bone excitation and response are markedly reduced and low-pass filtered when passing through soft tissues. In the last decade, the first limitation has been solved by progresses in electronics and ICT; cheap solutions for modal analysis are currently available also with advanced data processing. On the other side, as stressed by the authors in [Mattei et al., 2017], the damping effect can be overcome by means of pins screwed into the bone, i.e. in fractures treated with an external fixator. The placement of input and output instruments directly on the pins (and not on the skin) enable the transmission of more energy, also at higher frequencies, to the bone.

The present paper proposes the application of the procedure presented in [Mattei et al., 2017] to evaluate the progress of the healing process simulated in vitro. A Sawbone tibia phantom was fractured by an orthopedic surgeon and then the callus was artificially simulated with three different materials for describing its evolution. With respect to our previous paper, where the procedure was defined on an intact phantom, the present study is focused on the capability of the proposed approach to detect changes in the callus stiffness. This point deserves investigation because, while on the one hand, the use of pins screwed into the bone can help the vibration transmission to/from it, on the other 
hand, the introduction of additional elements may hide the response of the bone. Therefore, a test campaign was planned to investigate, in controlled conditions, whether and how the vibratory response of the phantom with fixator is affected by the evolution of the simulated callus. This study represents a second step of feasibility and reliability for the clinical application of the mechanical vibration approach to fracture healing assessment.

\section{Background on Fracture Healing and Callus Mechanical Properties}

Fracture healing is a complex and fascinating phenomenon, through which the bone is capable of self-repairing after a damage or a traumatic event. When a bone s fractured, the so-called secondary or indirect healing occurs. It is characterized by a callus formation that evolves through several phases: inflammation, callus differentiation, ossification and remodeling [Doblaré et al., 2004, Marsell and Einhorn, 2011].

The timescale of the healing process varies from patient to patient, depending on the subjective response as well as on the treatment, fracture type and fracture site [Ito and Perren]. For a long bone of the lower limb, inflammation covers approximately the first two-three weeks, in which the healing activates forming a hematoma, from which a granulation tissue develops. In the next stage (weeks 3-6), a fibro-cartilaginous tissue can be observed, named soft callus; it joins the fracture ends (union) providing them an initial stability. In weeks 6-12, the cartilage will be resorbed and replaced by a rather disorganized bony tissue, named hard callus, which provides stiffness and strength to the union [García-Rodríguez and Martínez-Reina, 2016]. Although this hard callus is rigid and has weight-bearing capacity, a few years can be necessary for the bone to restore completely its microstructure and original shape, including the medullary canal [Leong and Morgan, 2008, Wehner et al., 2010]. This last stage is known as bone remodeling. The healing process strongly depends on the mechanical actions on the callus, that determine the relative movement of the bone fragments, [Leong and Morgan, 2008, Steiner et al., 2014].

In external fixation, the phase of major interest of the healing process goes from week 6 to week 14 after fracture, for planning the device removal. This period corresponds to the evolution of the soft callus (SC) in hard callus (HC).

In order to simulate this evolution in-vitro, the mechanical properties of the callus in its different phases should be reproduced. While there are many studies in the literature on sheep and rat bone healing, e.g. [Floerkemeier et al., 2010, Mora-Macias et al., 2015], only few data can be found on the callus stiffness in humans, as observed in [Nikiforidis et al., 1990, Claes and Cunningham, 2009]. One of the most statistically relevant survey is focused on the evaluation of the tibia bending stiffness during healing, [Richardson et al., 1994]. Approximate values of the Young modulus of tissues can be found in numerical studies on bone healing simulation. In [Byrne et al., 2011] a value of $10 \mathrm{MPa}$ is assumed for the cartilage, $1 \mathrm{GPa}$ for immature bone, $6 \mathrm{GPa}$ for mature bone, growing up to $17 \mathrm{GPa}$ for cortical bone. The overall callus stiffness assumes intermediate values, being a mix of several components. Finally, in other studies where callus surrogates were 
used, the soft callus was simulated with a silicon rubber (having an elastic modulus in the order of tens of MPa) [Nikiforidis et al., 1990], while an epoxy resin was suggested for the more advanced phases (Araldite ${ }^{\circledR}$, elastic modulus about 2-3 GPa) [Nikiforidis et al., 1990, Nakatsuchi et al., 1996, Ong et al., 2016].

\section{Materials and Methods}

\subsection{Tibia phantom and external fixator}

A left tibia phantom (4th generation Sawbones, model \#3402) was used for the experimental tests, Fig. 1. The model reproduces a left tibia of large size, about $405 \mathrm{~mm}$ long, with a mass of $370 \mathrm{~g}$. The mechanical behavior of the phantom is comparable to cadaveric tibia [2016]; in particular, the cortical bone has an elastic modulus in the range 10-16 GPa while the cancellous bone 137-155 MPa.

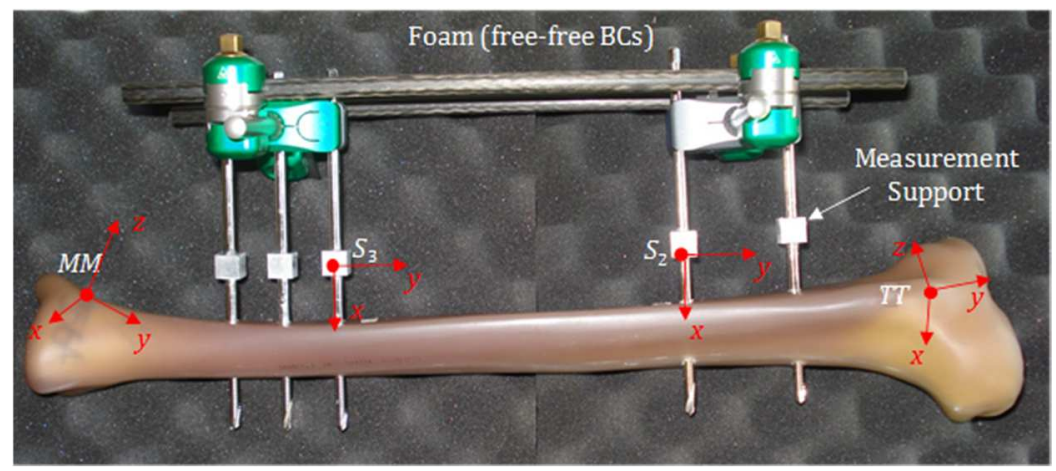

Fig. 1. Tibia phantom with five pins and Hoffman 2 fixator. Small cubed measurement supports (S2 and S3) can be observed on the pins. MM= medial malleolus, TT tibial tuberosity.

The Hoffmann 2 device by Stryker (mass $573 \mathrm{~g}$ ) with a bi-lateral frame in Vectran rods, was fixed to the bone by means of 5 self-drilling pins in stainless steel $(5 \mathrm{~mm}$ in diameter, $150 \mathrm{~mm}$ in length, $22 \mathrm{~g}$ in mass), as shown in Fig. 1. Small cubed supports were glued to the pins to assure a constant excitation direction and to ease accelerometer positioning. Measurement quality was thus improved. It can be worth underlying also that the fixator was assembled differently from what done in the previous study [Mattei et al., 2017].

\subsection{In- vitro callus simulation}

On the basis of the literature reported in $\S 2.2$, the evolution of the callus was simulated at different stages, by means of:

(1) A universal silicon by Bostik®;

(2) A glue based on HDPE by Pattex ${ }^{\circledR}$;

(3) Araldite ${ }^{\circledR}$. 
It is worth noting that the silicon and the Pattex ${ }^{\circledR}$ are initially semisolid whilst the Araldite ${ }^{\circledR}$ is a putty, whose hardening is achieved by handling it. In order to characterize such materials, some samples were preliminarily prepared and tested in compression. The estimated Young moduli were about $6 \mathrm{MPa}$ for the silicon a), $40 \mathrm{MPa}$ for the Pattex b), and $1 \mathrm{GPa}$ for Araldite c). Thus, they were used to simulate an initial soft callus (ISC), an advanced soft callus (SC) and a hard callus (HC), respectively.

The simulation of the callus started with a $4 \mathrm{~mm}$ thick osteotomy created by an orthopaedic surgeon in the middle of the phantom. The fixator was maintained assembled on the phantom for guaranteeing the relative position of the two parts (see Fig. 2(a)). Then, the glue was inserted to fill the gap and a proper hardening time was waited before performing measurements. The pictures of the artificial calluses are shown in Fig. 2(b-d). While $72 \mathrm{~h}$ were indicated in the datasheet for a complete solidification of Araldite ${ }^{\circledR}$, the callus surrogate appeared to be very hard already after a few hours. Thus, additional measurements were carried out on $\mathrm{PH}+\mathrm{P}$ after $20 \mathrm{~h}$ the putty positioning, simulating an initial hard callus, IHC.

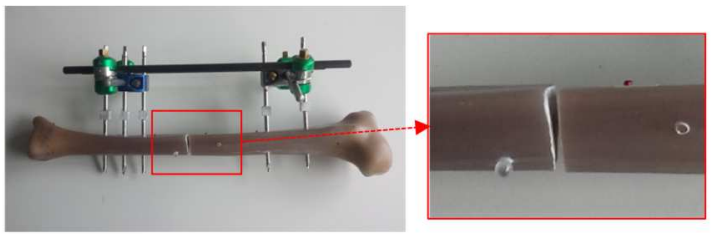

(a)

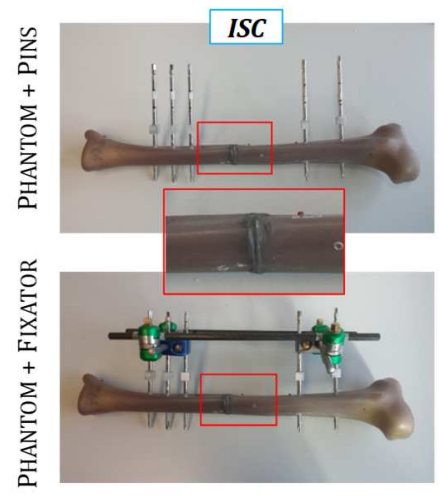

(b)

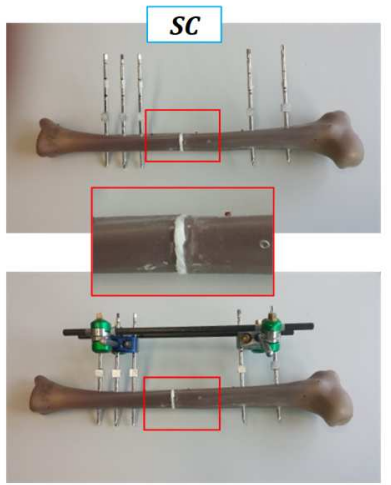

(c)

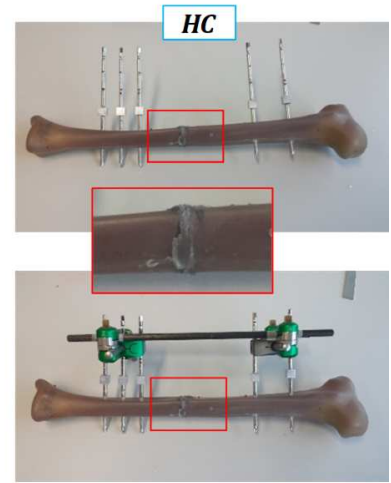

(d)

Fig. 2. Phantom with fracture (a) and phantom with artificial calluses in silicon (b), Pattex ${ }^{\circledR}(\mathrm{c})$ and Araldite ${ }^{\circledR}$ (d). The phantom with pins $(\mathrm{PH}+\mathrm{P})$ and phantom with complete fixator $(\mathrm{PH}+\mathrm{F})$ shown at the top and bottom of figures (b-d), respectively.

\subsection{Experimental set-up}

The impact test procedure defined in a previous study [Mattei et al., 2017] was employed in the test campaign. An instrumented micro-hammer (5800SL, Dytran $\left.{ }^{\circledR}\right)$ was used, having ultra-low mass of $9.8 \mathrm{~g}$ and very high stiffness. The output response was 
measured with a micro triaxial accelerometer (3133A1, Dytran $\left.{ }^{\circledR}\right)$, a $6.1 \mathrm{~mm}$ side cube with a mass of $0.8 \mathrm{~g}$. Tests were performed in free-free conditions, positioning the phantom on a rubber foam panel.

Data acquisition and data processing were performed using the LMS Test.Lab by Siemens ${ }^{\circledR}$. The Impact Testing module was used to optimize all test parameters and to perform tests, while the Modal Analysis module to analyze acquired data providing the power spectral density (PSD) of the excitation signal, the Frequency Response Functions (FRFs) and the coherence functions (CF). The resonant frequencies of the system were estimated by applying the Polymax curve fitting tool to the FRF sum function (i.e. the sum of the three FRFs obtained considering each accelerometer direction).

The frequency analysis was restricted to the bandwidth $0-1 \mathrm{kHz}$, according to the literature [Nikiforidis et al., 1990, Nakatsuchi et al., 1996, Bediz et al., 2010, Ong et al., 2016, Mattei et al., 2017]. All measurements were acquired in the frequency range 0$8192 \mathrm{~Hz}$, with a resolution of $2 \mathrm{~Hz}$. Each response was averaged over 10 trials.

\subsection{Test configurations}

Impact tests were performed on the tibia phantom with only pins $(\mathrm{PH}+\mathrm{P})$ and on the phantom with complete fixator $(\mathrm{PH}+\mathrm{F})$. In both cases, the phantom was in its original non-fractured conditions (XF, Fig.1) and fractured with the three types of calluses mentioned in $\S 3.2$ (ISC, SC, HC) and shown in Fig.2(b-d). The choice of examining the two systems, $\mathrm{PH}+\mathrm{P}$ and $\mathrm{PH}+\mathrm{F}$, moved from a practical reason. In [Mattei et al., 2017], it was proved that the bone response is determined more easily when only pins are present, than maintaining the whole fixator. This is because the pins simply add some concentrated mass and moment of inertia to the system, while the complete fixator also affects the stiffness markedly. In clinical practice, it could be possible to remove the fixator (but not the pins) about nine weeks after fracture, when monitoring the healing phase becomes more crucial. This configuration should be assumed for very short periods, while the leg is unloaded, e.g. laying in a bed or sitting in a high chair without touching the ground with the foot. The system $\mathrm{PH}+\mathrm{P}$ is thus preferred but in case clinical reasons discourage the fixator removal, the response of the $\mathrm{PH}+\mathrm{F}$ should be used. Concerns can arise about the repeatability of measurements after disassembling and reassembling the fixator, which was proved by the authors in [Mattei et al., 2017].

Another critical issue in impact tests is to properly locate input (hammer) and output (accelerometer) points and directions. Two solutions were considered: both points on the phantom or on the pins, respectively. In the first case, the tibial tuberosity (TT) and the medial malleolus $(M M)$ were considered, while in the second case points on the central pins, denoted $S_{2}$ and $S_{3}$ in Fig.1. The impact direction was defined by the local normal, i.e. $\mathrm{z}$-axis in Fig.1, while the accelerometer signals were acquired in the three $(x, y, z)$ directions. 


\section{Results}

\subsection{Phantom with pins}

Table 1 reports the resonant frequencies within the band $0-1 \mathrm{kHz}$ for different healing phases and input/output points. Each column of Table 1 refers to a specific mode that can occur at different frequencies depending on the callus type.

Tab.1. Resonant frequencies of the phantom with pins, non-fractured (XF) and at different phases of the simulated healing process (ISC: initial soft callus, SC: soft callus, IHC: initial hard callus, HC: hard callus), evaluated for different input/output configurations.

\begin{tabular}{|c|c|c|c|c|c|c|c|c|}
\hline $\begin{array}{l}\text { Input- } \\
\text { Output }\end{array}$ & $\begin{array}{c}\text { Healing } \\
\text { phase }\end{array}$ & $\mathbf{f}_{1}(\mathbf{H z})$ & $\mathbf{f}_{2}(\mathrm{~Hz})$ & $\mathbf{f}_{3}(\mathrm{~Hz})$ & $\mathbf{f}_{\mathbf{4}}(\mathbf{H z})$ & $\mathbf{f}_{5}(\mathrm{~Hz})$ & $\mathrm{f}_{6}(\mathrm{~Hz})$ & $f_{7}(H z)$ \\
\hline$X F$ & average & 244 & 276 & 294 & 390 & 464 & 520 & 844 \\
\hline \multirow{5}{*}{ ISC } & $M M-T T$ & 241 & 279 & & 331 & 388 & 425 & \\
\hline & $T T-M M$ & 239 & 278 & & 330 & 387 & 425 & \\
\hline & $S_{2}-S_{3}$ & 226 & 273 & & 335 & 390 & 426 & 683 \\
\hline & $S_{3}-S_{2}$ & 224 & 269 & & 335 & 390 & 424 & 683 \\
\hline & average & 233 & 275 & & 333 & 389 & 425 & 683 \\
\hline \multirow{5}{*}{$S C$} & $M M-T T$ & 233 & & 297 & 368 & 442 & 491 & 840 \\
\hline & $T T-M M$ & 244 & & 288 & 367 & 445 & 514 & 838 \\
\hline & $S_{2}-S_{3}$ & & & & 369 & 442 & 492 & 838 \\
\hline & $S_{3}-S_{2}$ & & & & 389 & 463 & 513 & 838 \\
\hline & average & 239 & & 293 & 373 & 448 & 503 & 839 \\
\hline \multirow{5}{*}{ IHC } & $M M-T T$ & 241 & & 295 & 389 & 460 & 513 & 850 \\
\hline & $T T-M M$ & 248 & & 288 & 389 & 460 & 514 & 849 \\
\hline & $S_{2}-S_{3}$ & & 274 & & 389 & 463 & 512 & 850 \\
\hline & $S_{3}-S_{2}$ & & & & 389 & 463 & 513 & 850 \\
\hline & average & 245 & 274 & 292 & 389 & 462 & 513 & 850 \\
\hline \multirow{5}{*}{$H C$} & $M M-T T$ & 243 & & 299 & 391 & 463 & 522 & 856 \\
\hline & $T T-M M$ & 245 & & 299 & 390 & 462 & 517 & 853 \\
\hline & $S_{2}-S_{3}$ & & & & 392 & 467 & 516 & 855 \\
\hline & $S_{3}-S_{2}$ & & 272 & & 391 & & 516 & 856 \\
\hline & average & 244 & 272 & 299 & 391 & 464 & 518 & 855 \\
\hline
\end{tabular}

A good repeatability of all measurements can be observed. The quality of the measurements was confirmed by the good coherence functions, generally close to unit, while the measurements reciprocity was verified by comparing the FRFs for TT-MM vs. MM-TT tests, and $S_{2}-S_{3}$ vs. $S_{3}-S_{2}$. Comparing the resonant frequencies estimated from different input/output points, negligible discrepancies were obtained for all the types of callus. However, the phantom-phantom combination (TT-MM) allowed to identify a 
higher number of modes with respect to pin-pin one $\left(S_{2}-S_{3}\right)$, in the SC, IHC and HC conditions, exciting also resonant frequencies lower than $400 \mathrm{~Hz}$. The opposite was observed for the ISC, as the highest resonant frequency $\left(f_{7}\right)$ could not be evaluated in the TT-MM configuration.

The non-fractured phantom showed seven modes in the bandwidth $0-1 \mathrm{kHz}$, while the ISC only six modes, missing the one at $294 \mathrm{~Hz}$. Additionally, in the ISC case the resonant frequencies were lower than those of the XF case, with percentage variations from $5 \%$ for $f_{l}$ to $19 \%$ for $f_{7}$. This reduction was expected, as the silicon used to simulate a granular tissue was much softer than the cortical bone. A damping effect of the silicon was also observed from the lower and wider peaks with respect to the non-fractured case, as shown in Fig. 3 (a,b). The FRFs of the XF and ISC phantoms for both $S_{2}-S_{3}$ and MM-TT configurations are compared in Fig. 3(a,b): the two trends looks very different as the FRF of the ISC specimen having only one peak at frequencies higher than $450 \mathrm{~Hz}$.

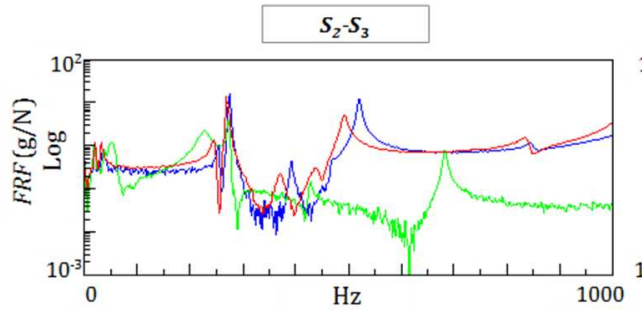

(a)

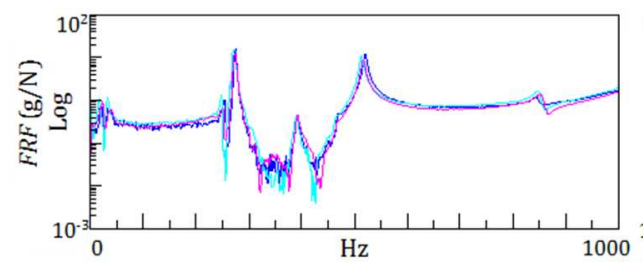

(c)

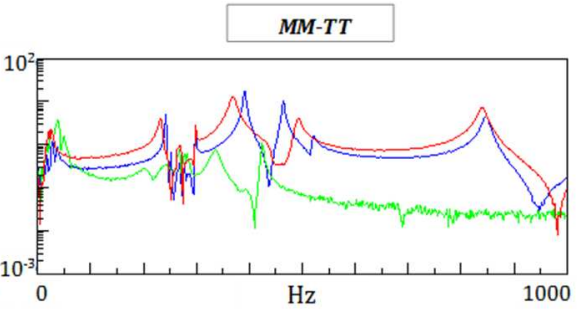

(b)

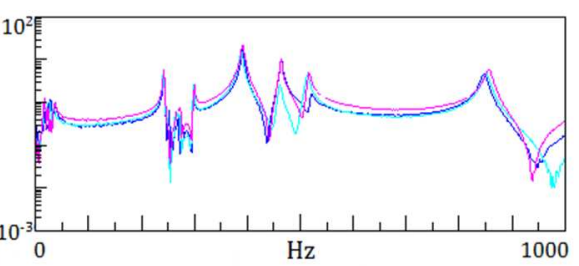

(d)

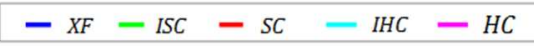

Fig. 3. Simulation of bone healing process for the $\mathrm{PH}+\mathrm{P}$ system. Comparison of FRFs of the non-fractured (XF) vs. fractured phantom at different phases of healing process (ISC, SC, IHC and HC) obtained with two inputoutput configurations: $S_{2}-S_{3}$ (output in z) on the left (a,c), and $M M-T T$ (output in x) on the right (b,d).

For the SC case, six modes were identified, including the third mode of the intact phantom but not the second one. Also in this case, all resonant frequencies were lower than those of the XF phantom, though the percentage deviations were lower than for the ISC specimen (in particular lower than 4\%). In Fig. 3(c,d), the FRFs of SC and XF cases show pretty similar trends for all configurations indicating a progress in the healing process with respect to the ISC phantom. As far as the late phase of healing is concerned, both in the IHC and $\mathrm{HC}$ conditions, $\mathrm{PH}+\mathrm{P}$ response was characterized by seven modes 
almost equal to those of the non-fractured phantom (percentage variations $<1 \%$ ). The very similar dynamic behavior of the XF, IHC and HC specimens was confirmed by almost equal FRFs portrayed in Fig. 3 (c,d). Although IHC and HC were obtained using Araldite ${ }^{\circledR}$ at different hardening times, $20 \mathrm{~h}$ and $72 \mathrm{~h}$ respectively, results were very similar, indicating that at $20 \mathrm{~h}$ a complete glue hardening was already achieved. Thus, only the $\mathrm{HC}$ case will be considered in the following. Moreover, the likeness of the XF and $\mathrm{HC}$ curves proved that Araldite ${ }^{\circledR}$ simulated properly a healed fracture.

Results demonstrated that the vibration response significantly changes with different types of callus and thus can be a useful tool for monitoring the healing process.

\section{Phantom with fixator}

The resonant frequencies estimated for the $\mathrm{PH}+\mathrm{F}$ system are collected in Table 2. Each column of Table 2 identifies the same mode for the XF, SC and HC specimens. Differently, the resonant frequencies of ISC are reported without considering possible correlations with modes of other cases, as discussed later.

A good repeatability of measurements was demonstrated for all specimens by negligible variations of the resonant frequencies estimated using different test configurations $(<2 \%)$. The reciprocity of system was also preliminarily verified since the numerous clamps and screwed connections of the fixator were thought to compromise the system linearity. As for the $\mathrm{PH}+\mathrm{P}$ system, different modes were excited by different test configurations. The combinations $M M-T T$ and $S_{2}-S_{3}$ were capable of exciting the largest number of modes and thus were considered for further analyses.

The vibrational analysis of ISC revealed 14 resonant frequencies (see Table 2), as for the non-fractured phantom with fixator. As mentioned above, a correlation between the modes of the two specimens was difficult to find out, because their FRFs were very different (Fig. 4(a,b)), both for $M M-T T$ and $S_{2}-S_{3}$ configurations. Comparing such FRFs, only few modes can be matched, e.g. $f_{1}, f_{2}$ and $f_{5}$, with lower values for the ISC than for the $\mathrm{XF}$, which is agreement with the previous results for the $\mathrm{PH}+\mathrm{P}$ system. The silicon callus also demonstrated a certain damping effect.

The SC case was characterized by 10 resonant frequencies (Table 2). Similar trends of the FRFs of the SC and the XF phantom can be observed in shown in Fig. 4(a,b), indicating an initial consolidation of the bone. A comparison of the SC and XF modes highlighted that out of the 14 modes of the XF phantom, the fourth and the highest ones were not present for the SC case. Some resonant frequencies estimated for the SC, i.e. $f_{l}$, $f_{5}-f_{8}$, were lower than the XF ones, according to the fact that the SC callus is softer than the cortical bone, with percentage variations of the average values down to $12 \%$ (for $f_{6}$ ). This effect can also be observed in Fig. 4(a,b), where some peaks of FRFs for the SC case are shifted towards the low frequencies with respect to the correspondent ones of the XF specimen.

Finally, the HC case presented 14 resonant frequencies (Table 2), as the non-fractured sample. Their dynamic behaviour was very similar both in terms of values of resonant frequencies (differences lower than 4\%) and FRFs trends, depicted in Fig. 4(c,d). The 
resonant frequencies of the $\mathrm{HC}$ specimen were slightly higher than the XF ones, particularly above $500 \mathrm{~Hz}$.

Results demonstrated that bone healing can be monitored also in presence of fixator though it complicates the FRFs and increases the number of resonant frequencies to be considered in the analysis.

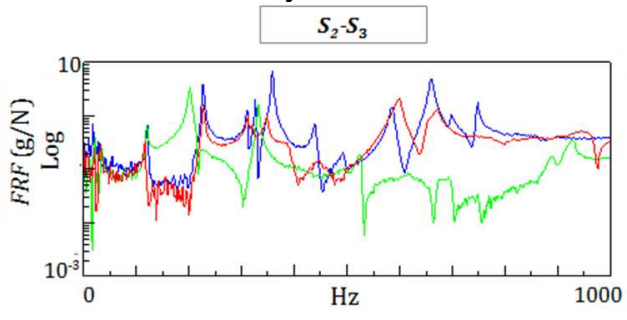

(a)

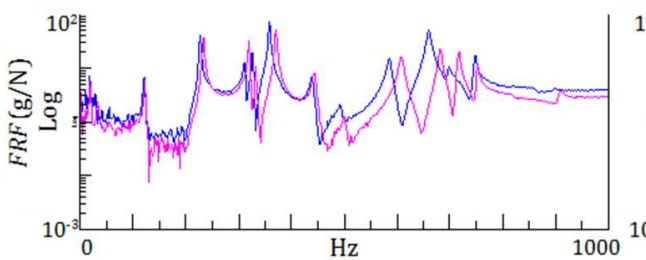

(c)

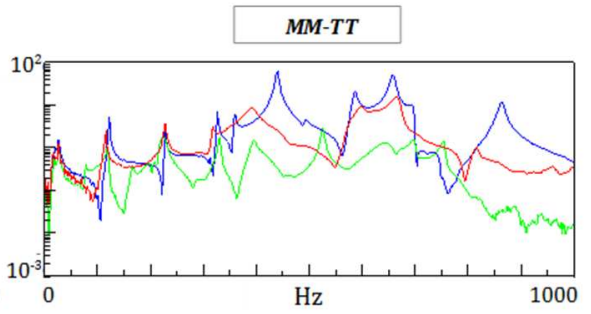

(b)

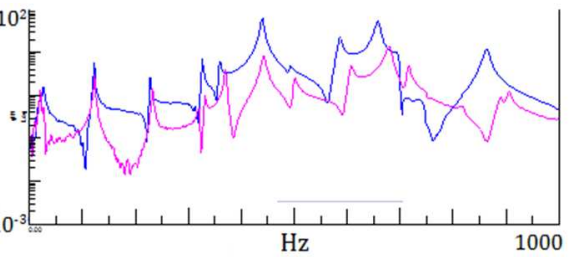

(d)

$-X F-I S C-S C-I H C-H C$

Fig. 4. Simulation of bone healing in PH+F specimens. Comparison of FRFs of the non-fractured (XF) vs. fractured phantom at different phases of healing process (ISC, SC, IHC and HC) obtained with two input-output configurations: $S_{2}-S_{3}$ (output in z) on the left (a,c), and $M M-T T$ (output in x) on the right (b,d).

Tab.2 Resonant frequencies of the phantom with fixator, non-fractured (XF) and at different phases of the simulated healing process (ISC, SC, IHC and HC), evaluated for different input/output configurations.

\begin{tabular}{|c|c|c|c|c|c|c|c|c|c|c|c|c|c|c|c|}
\hline $\begin{array}{l}\text { Input- } \\
\text { Output }\end{array}$ & $\begin{array}{c}\text { Healing } \\
\text { phase }\end{array}$ & $\mathrm{f}_{1}(\mathrm{~Hz})$ & $\mathrm{f}_{2}(\mathrm{~Hz})$ & $\mathrm{f}_{3}(\mathrm{~Hz})$ & $\mathbf{f}_{4}(\mathrm{~Hz})$ & $f_{5}(\mathrm{~Hz})$ & $f_{6}(\mathrm{~Hz})$ & $f_{7}(\mathrm{~Hz})$ & $f_{8}(\mathrm{~Hz})$ & $f_{9}(\mathrm{~Hz})$ & $f_{10}(\mathrm{~Hz})$ & $f_{11}(\mathrm{~Hz})$ & $f_{12}(\mathrm{~Hz})$ & $\mathrm{f}_{13}(\mathrm{~Hz})$ & $\mathrm{f}_{14}(\mathrm{~Hz})$ \\
\hline \multirow{5}{*}{$X F$} & $M M-T T$ & 121 & 227 & 312 & 326 & 359 & 439 & 491 & 585 & 657 & 695 & 745 & 817 & 863 & \\
\hline & $T T-M M$ & 120 & 226 & 311 & 326 & 358 & & 489 & 583 & 659 & 698 & & 819 & 864 & 894 \\
\hline & $S_{2}-S_{3}$ & 122 & 227 & 311 & 326 & 359 & 439 & 492 & 585 & 660 & 697 & 746 & 818 & 864 & 896 \\
\hline & $S_{3}-S_{2}$ & 122 & 227 & & & 359 & 439 & 494 & 586 & 660 & 697 & 746 & 818 & 864 & \\
\hline & average & 121 & 227 & 311 & 326 & 359 & 439 & 492 & 585 & 659 & 697 & 746 & 818 & 864 & 895 \\
\hline \multirow{5}{*}{$S C$} & $M M-T T$ & 116 & & 224 & & 329 & & 396 & 525 & 632 & & 695 & 750 & & 936 \\
\hline & $T T-M M$ & 118 & 201 & 224 & 285 & 331 & & 398 & 510 & & 662 & 694 & 750 & 888 & 928 \\
\hline & $S_{2}-S_{3}$ & 120 & 201 & 218 & 283 & 331 & & 391 & 527 & 646 & 670 & 690 & & 887 & 924 \\
\hline & $S_{3}-S_{2}$ & 119 & 200 & & & 330 & & 395 & 525 & & & 693 & 750 & 892 & 923 \\
\hline & average & 118 & 201 & 222 & 284 & 330 & & 395 & 522 & 639 & 666 & 693 & 750 & 889 & 928 \\
\hline \multirow{5}{*}{ ISC } & $M M-T T$ & 114 & 227 & 313 & & 360 & 390 & 447 & 594 & 664 & & & 816 & & 960 \\
\hline & $T T-M M$ & 115 & 227 & 315 & & 350 & 389 & 450 & & 665 & & & 815 & & 970 \\
\hline & $S_{2}-S_{3}$ & & 227 & 314 & & 344 & 389 & 454 & 597 & 662 & & & 804 & & 969 \\
\hline & $S_{3}-S_{2}$ & & 226 & 314 & & 347 & 385 & 448 & 591 & 670 & & & 811 & & 962 \\
\hline & average & 115 & 227 & 314 & & 350 & 388 & 450 & 594 & 665 & & & 812 & & 965 \\
\hline \multirow{5}{*}{$H C$} & $M M-T T$ & 122 & 232 & 320 & 331 & 370 & 442 & 500 & 606 & 679 & 714 & 749 & 817 & 890 & 903 \\
\hline & $T T-M M$ & 122 & 233 & 320 & & 370 & 442 & 498 & 606 & 680 & 716 & 752 & 821 & 892 & 906 \\
\hline & $S_{2}-S_{3}$ & 123 & 233 & 319 & 331 & 370 & 443 & 500 & 608 & 682 & 716 & 751 & 820 & & 906 \\
\hline & $S_{3}-S_{2}$ & 122 & 233 & 319 & 332 & 370 & 443 & 501 & 608 & 681 & 716 & 750 & 820 & 888 & 905 \\
\hline & average & 122 & 233 & 320 & 331 & 370 & 443 & 500 & 607 & 681 & 716 & 751 & 820 & 890 & 905 \\
\hline
\end{tabular}




\section{Discussion and Conclusions}

In a previous study, the authors defined a procedure based on impact testing that could be useful for fracture healing assessment in cases treated with an external fixator [Mattei et al., 2016]. An intact Sawbone tibia phantom was used for tests, carrying a Hoffmann 2 device assembled on the phantom through five pins screwed into it. The aim of that study was to find the best solution for extracting the resonant frequencies of the systems $\mathrm{PH}+\mathrm{P}$ and $\mathrm{PH}+\mathrm{F}$.

In this study, the tibial phantom was fractured and the healing process was simulated by creating artificial calluses mimicking different healing phases. The external fixator and the pins were maintained. The vibratory response of the phantom with complete fixator $(\mathrm{PH}+\mathrm{F})$ or only with the pins $(\mathrm{PH}+\mathrm{P})$ was experimentally investigated through impact tests, according to the procedure defined in [Mattei et al., 2016]. The aim of this study was to verify the reliability of the mechanical vibration approach to fracture healing assessment. The capability of the measurement setup and data processing to detect variations in the callus stiffness was thus to be proved. For ethical reasons, we preferred to avoid tests on animals and to pursue an in vitro validation before moving to human patients.

The repeatability of measurements and the reciprocity of both systems $(\mathrm{PH}+\mathrm{P}$ and $\mathrm{PH}+\mathrm{F})$ were preliminarily verified. The proper choice of the input (hammer) and output (accelerometer) points was a crucial issue for the identification of all the vibratory modes of the systems. In all cases, best results were obtained when pins were used both for exciting the bone and capturing its response. This can be due to the presence of supports glued on the pins that ensured a constant excitation direction.

As far as the simulation of the healing process is concerned, the experimental modal analysis highlighted a remarkable difference between the vibratory response of the early phase sample (ISC) and of the more advanced ones (SC, IHC, HC). A trend towards a non-fractured condition (XF) was also observed. In the ISC sample, the silicon callus was really very soft and introduced also a marked damping effect, thus the FRF is very different from the other cases. As the stiffness increases from SC to HC samples, the FRFs become more similar and the resonant frequencies are shifted towards higher values, as expected. This is in agreement with both ex vivo and in vitro studies, [Nakatsuchi et al., 1996] and [Ong e al. et al., 2016]. These results can be considered encouraging and provide a satisfactory validation of the approach. It was also confirmed that leaving only the pins screwed into the bone, but removing the fixator, should be preferred for tests, as the response of the underlying bone is more evident.

The simulation of the callus as a homogeneous cylinder is a limitation of the study. As other researchers in the literature, we preferred to have a simple geometry with a quantifiable stiffness, then to complicate the callus replica loosing the control of its mechanical response. The elastic modulus of the calluses was preliminarily estimated by 
means of compression tests at a rather low strain rate. Since the response of these artificial materials is dependent on the strain rate, it is reasonable to expect that at high frequencies the elastic moduli will be higher than reported. The obtained values can thus be considered as semi-qualitative indications of the material responses, to be used in comparative terms among the different phases. It is worth reminding that no data on human calluses are available in the literature to assess their accuracy. In real cases, the fractured region is usually rather wide, larger than the $4 \mathrm{~mm}$ used in the tests. The overall stiffness of the callus should therefore be combined with a geometrical effect that can be helpful for detecting the evolution of the healing process.

There are no similar studies in the literature for a comparative discussion of results. Some critical points and limits for the clinical application of modal analysis to healing assessment can be worth mentioning, that are currently under investigation in in-vivo tests. The first crucial issue concerns the hypothesis that a shift in the resonant frequencies can be ascribed to a change in the stiffness of the bone. This is not completely true as it could also be due to a variation in the mass of the segment, e.g. muscle mass, or an increase in the muscle tone. A reduction in the mass can determine an increase in the resonant frequencies, which could be confused with a stiffening of the callus. It will be important to verify in vivo whether it will be possible or not to differentiate between these cases. It is expected that in few weeks during the healing process, the bone stiffness will change more than the muscle mass. The posture of the patient could also affect measurements and should be investigated, with the aim of keeping muscles as relaxed as possible.

In conclusion, the present study demonstrated the feasibility of bone healing assessment by means of vibrational analysis, for fractures treated with an external fixator. A change in the callus stiffness during healing causes differences in the dynamic response of the structure both with and without fixator (i.e. leaving only pins screwed into the bone), which can be detected through experimental modal analysis. Monitoring the healing evolution is easier without the fixator than with it, as its complex structure and connections complicate the FRFs and cause a high number of close resonant frequencies, more difficult to interpret.

In both cases, $\mathrm{PH}+\mathrm{P}$ and $\mathrm{PH}+\mathrm{F}$, the pins screwed into the bone are a key point for measurement quality since they allow to overcome the damping of the soft tissues. While the application of mechanical vibrations to bone healing assessment is not new in the literature, the exploitation of the pins is a novel suggestion from our studies. It can enhance the feasibility and reliability to the proposed approach.

The present procedure is currently applied in two clinical cases.

\section{Acknowledgments}

This work was supported by the University of Pisa, (PRA 2017).

\section{References}

"LMS PolyMAX. A Revolution in Modal Parameter Estimation." 
[2016] "Biomechanical test materials," www. sawbones.com.

Bediz, B., Nevzat Ozguven, H. and Korkusuz, F. [2010] "Vibration measurements predict the mechanical properties of human tibia," Clin Biomech 25(4), 365-371.

Bhandari, M., Guyatt, G. H., Swiontkowski, et al. [2001] "Surgeons' preferences for the operative treatment of fractures of the tibial shaft. An international survey," J Bone Joint Surg Am 83-A(11), 1746-1752.

Byrne, D. P., Lacroix, D. and Prendergast, P. J. [2011] "Simulation of fracture healing in the tibia: mechanoregulation of cell activity using a lattice modeling approach," J Orthop Res 29(10), 14961503.

Checketts, R. G. and Young, C. F. [2003] "External fixation of diaphyseal fractures of the tibia," Current Orthopaedics 17(3), 176-189.

Claes, L. E. and Cunningham, J. L. [2009] "Monitoring the mechanical properties of healing bone," Clinical Orthopedics and Related Research 467(8), 1964-1971.

Cunningham, J. L., Kenwright, J. and Kershaw, C. J. [1990] "Biomechanical measurement of fracture healing," J Med Eng Technol 14(3), 92-101.

Davies, R., Holt, N. and Nayagam, S. [2005] "The care of pin sites with external fixation," Journal of Bone \&amp; Joint Surgery, British Volume 87-B(5), 716-719.

Doblaré, M., García, J. M. and Gómez, M. J. [2004] "Modelling bone tissue fracture and healing: a review," Engineering Fracture Mechanics 71(13-14), 1809-1840.

Eastaugh-Waring, S. J., Hardy, J. R. W. and Cunningham, J. L. [2000] "Fracture stiffness measurement using the orthometer: reproducibility and sources of error," Clinical Biomechanics 15(2), 140-142.

Floerkemeier, T., Thorey, F., Hurschler, C., Wellmann, M., Witte, F. and Windhagen, H. [2010] "Stiffness of callus tissue during distraction osteogenesis," Orthop Traumatol Surg Res 96(2), 155160.

García-Rodríguez, J. and Martínez-Reina, J. [2016] "Elastic properties of woven bone: effect of mineral content and collagen fibrils orientation," Biomechanics and modeling in mechanobiology, $1-14$.

Ito, K. and Perren, S. M. "Biology of fracture healing," www2.aofoundation.org/wps/portal/surgerymobile?contentUrl=/srg/popup/further_reading/PFxM 2/12_33_biol_fx_heal.jsp\&soloState $=$ precomp $\&$ title $=\&$.

Leong, P. L. and Morgan, E. F. [2008] "Measurement of fracture callus material properties via nanoindentation," Acta Biomater 4(5), 1569-1575.

Li, H., Le, L. H., Sacchi, M. D. and Lou, E. H. [2013] "Ultrasound imaging of long bone fractures and healing with the split-step fourier imaging method," Ultrasound Med Biol 39(8), 1482-1490.

Markel, M. D. and Chao, E. Y. [1993] "Noninvasive monitoring techniques for quantitative description of callus mineral content and mechanical properties," Clinical Orthopedics and Related Research(293), 37-45.

Marsell, R. and Einhorn, T. A. [2011] "The biology of fracture healing," Injury 42(6), 551-555.

Mattei, L., Longo, A., Di Puccio, F., Ciulli, E. and Marchetti, S. [2017] "Vibration Testing Procedures for Bone Stiffness Assessment in Fractures Treated with External Fixation," Annals of biomedical engineering 45(2), 1111-1121.

Mora-Macias, J., Reina-Romo, E., Lopez-Pliego, M., Giraldez-Sanchez, M. A. and Dominguez, J. [2015] "In Vivo Mechanical Characterization of the Distraction Callus During Bone Consolidation," Ann Biomed Eng 43(11), 2663-2674.

Nakatsuchi, Y., Tsuchikane, A. and Nomura, A. [1996] "The vibrational mode of the tibia and assessment of bone union in experimental fracture healing using the impulse response method," Medical Engineering \& Physics 18(7), 575-583.

Nikiforidis, G., Bezerianos, A., Dimarogonas, A. and Sutherland, C. [1990] "Monitoring of fracture healing by lateral and axial vibration analysis," J Biomech 23(4), 323-330. 
Ong, W. H., Chiu, W. K., Russ, M. and Chiu, Z. K. [2016] "Integrating sensing elements on external fixators for healing assessment of fractured femur," Structural Control and Health Monitoring, in press DOI: 10.1002/stc.1843, 2016.

Pontarelli, W. R. [1982] "External Fixation of Tibial Fractures," The Iowa Orthopaedic Journal 2, 80-88.

Richardson, J. B., Cunningham, J. L., Goodship, A. E., Connor, B. T. and Kenwright, J. [1994] "Measuring stiffness can define healing of tibial fractures," Journal of Bone \& Joint Surgery, Series B 76(3), 389-394.

Steiner, M., Claes, L., Ignatius, A., Simon, U. and Wehner, T. [2014] "Numerical simulation of callus healing for optimization of fracture fixation stiffness," Plos One 9(7), e101370.

Wehner, T., Claes, L., Niemeyer, F., Nolte, D. and Simon, U. [2010] "Influence of the fixation stability on the healing time--a numerical study of a patient-specific fracture healing process," Clin Biomech 25(6), 606-612. 